\title{
Left ventricular assist device implantation after acute anterior wall myocardial infarction and cardiogenic shock: A two-center study
}

Nicholas C. Dang, MD, ${ }^{\text {a }}$ Veli K. Topkara, MD, ${ }^{\mathrm{a}}$ Marzia Leacche, MD, ${ }^{\mathrm{b}}$ Ranjit John, MD, ${ }^{\text {a }}$ John G. Byrne, MD, ${ }^{\mathrm{b}}$ and Yoshifumi Naka, MD, PhD

From the Division of Cardiothoracic Surgery, Department of Surgery, ${ }^{\text {a }}$ Columbia University, College of Physicians and Surgeons, New York, NY, and the Division of Cardiac Surgery, Department of Surgery, ${ }^{\mathrm{b}}$ Brigham \& Women's Hospital, Boston, Mass.

Dr Yoshifumi Naka is Herbert Irving Assistant Professor of Surgery at Columbia University, College of Physicians and Surgeons. Dr John G. Byrne is William S. Stoney Professor of Surgery and Chairman, Department of Cardiac Surgery, Vanderbilt University Medical Center.

Received for publication Dec 7, 2004; revisions received March 25, 2005; accepted for publication April 12, 2005.

Address for reprints: Yoshifumi Naka, MD, $\mathrm{PhD}$, Herbert Irving Assistant Professor of Surgery, Division of Cardiothoracic Surgery, Department of Surgery, Columbia University, College of Physicians and Surgeons, 177 Fort Washington Ave, Milstein Hospital, 7GN-435, New York, NY 10032 (E-mail: yn33@columbia.edu).

J Thorac Cardiovasc Surg 2005;130:693-8

$0022-5223 / \$ 30.00$

Copyright (c) 2005 by The American Association for Thoracic Surgery

doi:10.1016/j.jtcvs.2005.04.014
Objective: Left ventricular assist device (LVAD) insertion after anterior wall myocardial infarction complicated by cardiogenic shock is an accepted modality of support in select patients. Results of primary revascularization for these patients are poor. We seek to determine the outcomes of patients with myocardial infarction and shock who undergo LVAD insertion alone versus surgical revascularization before LVAD insertion.

Methods: Seventy-four patients at 2 institutions underwent LVAD implantation for myocardial infarction and shock over a 12 -year period. Twenty-eight underwent direct LVAD placement, and 46 underwent revascularization through coronary artery bypass grafting before LVAD placement. Variables examined included patient demographics, myocardial infarction-LVAD interval, bridge to transplantation, early mortality ( $\leq 30$ days), survival after LVAD placement, and posttransplantation survivals.

Results: There were no differences in demographics between the 2 groups. The group undergoing revascularization before LVAD placement had a lower bridge to transplantation, higher early mortality, and lower overall 6- and 12-month survivals after LVAD placement compared with the group undergoing direct LVAD placement $(45.50 \%$ vs $70.40 \%, P=.041 ; 39.10 \%$ vs $14.30 \%, P=.020 ; 89.3 \%$ and $82.1 \%$ vs $54.4 \%$ and $52.2 \%$, respectively, $P=.006$ ). Posttransplantation survival and LVAD explantation rates were equivalent in both groups.

Conclusions: Coronary artery bypass grafting before LVAD insertion for cardiogenic shock complicating myocardial infarction adversely affects survival. Confirmation of these findings would require conducting a large, multicenter, randomized clinical trial comparing revascularization versus LVAD support as primary therapy in this setting.

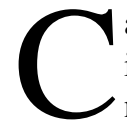
ardiogenic shock (CS) complicates $5 \%$ to $10 \%$ of cases of acute myocardial infarction (MI) and has a mortality rate of up to $80 \%$ by 1 year. ${ }^{1}$ Percutaneous coronary intervention (PCI; transluminal coronary angioplasty-stent placement) and coronary artery bypass graft (CABG) surgery represent strategies to revascularize the ischemic myocardium subserved by the infarcted vessel. Both of these approaches focus on the acute mechanism of MI and attempt to restore adequate coronary blood flow.

Previous studies have reported lower mortality rates for patients with acute MI and CS who subsequently undergo either PCI or CABG procedures compared with rates for patients treated with conventional therapy alone (no revascularization). ${ }^{2-8}$ Furthermore, survival seems to be contingent on the success of restoration of blood flow within the infarcted vessel., $3,7,9$ Short-term survival for these cohorts of patients ranges from $47 \%$ to $65 \%$. These results, although satisfactory compared 
with those of their medically treated counterparts, remain grim. We have considered whether such revascularization strategies alone might be insufficient to reverse the clinical condition of shock in these patients and whether a greater level of cardiac and circulatory support is necessary.

The use of left ventricular assist devices (LVADs) for the treatment of end-stage heart failure is well established, both in terms of bridging patients to cardiac transplantation and providing long-term support as a means of destination therapy. ${ }^{10}$ Less clearly defined is the role of LVADs in the setting of acute MI complicated by CS. Early reports show modest success with this modality of support, and patients are either weaned from LVADs or effectively bridged to cardiac transplantation. ${ }^{11-15}$ With improving clinical outcomes of LVADs, their role as primary therapy for acute MI complicated by CS needs to be evaluated.

\section{Methods}

This study protocol was approved by the respective institutional review boards of Columbia University, the College of Physicians and Surgeons (New York, NY), and Brigham \& Women's Hospital (Boston, Mass). Data were retrospectively analyzed after databases were stripped of all patient identifiers and a unique code number was used for each study subject.

Acute MI was defined as that having occurred 3 months or less before LVAD implantation and was manifest by ST-segment elevation of greater than $1 \mathrm{~mm}$ on electrocardiography, increased cardiac enzyme levels (creatine kinase MB fraction, troponin, lactic dehydrogenase, and myoglobin), and written documentation of diagnosis by an attending cardiologist. The broad definition of acute MI was established to ensure thorough inclusion and characterization of all patients who received LVADs as a direct result of acute MI and CS. This time interval also accommodated for the additional time that might have elapsed for patients who first underwent revascularization. CS was confirmed by both clinical and hemodynamic criteria and was required to be a direct result of the acute MI. Clinical criteria included systolic blood pressure of less than $90 \mathrm{~mm} \mathrm{Hg}$ or the need for intravenous vasopressors to maintain a systolic blood pressure of $90 \mathrm{~mm} \mathrm{Hg}$ or greater, as well as evidence of end-organ hypoperfusion, such as diminished urinary output $(<30 \mathrm{~mL} / \mathrm{h})$ or cool extremities. Hemodynamic criteria included a documented cardiac index of less than 2.2 $\mathrm{L} \cdot \mathrm{min}^{-1} \cdot \mathrm{m}^{-2}$ and pulmonary artery diastolic pressure or pulmonary capillary wedge pressure of $15 \mathrm{~mm} \mathrm{Hg}$ or greater.

Of all the patients who received LVADs at the 2 institutions from October 1991 through December 2003, 74 were identified who had acute anterior wall MI and CS as their primary indication. All patients with diagnoses of chronic congestive heart failure and nonanterior wall MIs were excluded to eliminate alternate possible indications for LVAD support. Six patients received ABIOMED (ABIOMED, Inc, Danvers, Mass) BVS 5000 biventricular support systems, 5 received ABIOMED BVS 5000 LVADs, 1 received a Thoratec (Thoratec Corp, Pleasanton, Calif) paracorporeal LVAD, 9 received HeartMate (Thoratec Corp) implantable pneumatic LVADs, and the remaining 53 received HeartMate vented-electric LVADs. Twenty-eight patients underwent direct LVAD implantation after acute anterior wall MI and CS (LVAD), and 46 under- went attempted revascularization by means of CABG before eventually requiring LVAD support (CABG plus LVAD).

The 2 groups were compared in regard to patient demographics, time interval between diagnosis of acute MI and LVAD insertion (in days), and preoperative intra-aortic balloon pump use. Outcome variables included bridge-to-transplantation rate, LVAD explantation rate, 6- and 12-month survivals after LVAD placement (combined bridge to transplantation, LVAD explantation, and ongoing LVAD support rates), early mortality ( $\leq 30$ day) rate, overall mortality, total hospital length of stay (LOS), intensive care unit (ICU) LOS, and posttransplantation survival.

A second comparison was made between those patients receiving LVADs for 7 days or less (acute) and 7 days or more (subacute) after acute MI and CS, irrespective of revascularization status. Similar preoperative and clinical outcome variables were analyzed.

Data were represented as frequency distributions and simple percentages. Values of continuous variables were expressed as means \pm standard deviation. Continuous variables were compared by independent samples $t$ tests, and categorical variables were compared by $\chi^{2}$ and Fisher exact tests, where appropriate. Kaplan-Meier analysis was used to calculate long-term survival with a $\log$-rank $P$ value when comparing groups. Actuarial survival was calculated by constructing life tables. All data were analyzed with SPSS version 11.5 software (SPSS, Inc, Chicago, Ill).

\section{Results \\ Demographics}

The clinical characteristics of patients with LVADs according to group (LVAD vs CABG plus LVAD and acute versus subacute) are outlined in Table 1 . The overall mean age was $53.6 \pm 8.8$ years. Fifty-eight $(78.4 \%)$ patients were male, and $16(21.6 \%)$ were female. Mean and median acute MILVAD intervals were $9.7 \pm 13.6$ days and 5.0 days, respectively, for the entire study cohort but were otherwise equivalent between the LVAD and CABG plus LVAD groups. In the CABG plus LVAD group, the mean time interval between diagnosis of acute $\mathrm{MI}$ and $\mathrm{CABG}$ was $5.1 \pm 7.8$ days, and the mean time interval between CABG and LVAD implantation was $3.8 \pm 8.0$ days. Three patients were still receiving $L V A D$ support at the time of data analysis ( 1 in the LVAD group and 2 in the CABG plus LVAD group) and were therefore censored from calculation of transplantation and explantation rates.

\section{Bridge-to-Transplantation Success and LVAD Explantation}

The bridge-to-transplantation success rate was significantly higher in the LVAD group compared with that in the CABG plus LVAD group $(P=.041$, Figure 1$)$. LVAD explantation rates were similar between the LVAD and CABG plus LVAD groups and between the acute and subacute MILVAD interval groups (Figures 1 and 2). The overall success rate to either transplantation or explantation was significantly higher in the acute MI-LVAD interval group 
TABLE 1. Clinical characteristics of patients receiving LVADs

\begin{tabular}{|c|c|c|c|c|c|c|}
\hline & LVAD & CABG plus LVAD & $P$ value & Acute & Subacute & $P$ value \\
\hline Age (y) & $55.6 \pm 9.4$ & $52.4 \pm 8.3$ & .129 & $51.5 \pm 9.4$ & $57.4 \pm 6.0$ & .005 \\
\hline \multicolumn{7}{|l|}{ Sex } \\
\hline Male & $20(71.4 \%)$ & $38(82.6 \%)$ & .257 & $36(76.6 \%)$ & $22(81.5 \%)$ & .623 \\
\hline Female & $8(28.6 \%)$ & $8(17.4 \%)$ & & $11(23.4 \%)$ & $5(18.5 \%)$ & \\
\hline \multicolumn{7}{|l|}{ Race } \\
\hline White & $26(92.8 \%)$ & $37(82.2 \%)$ & .199 & $39(84.8 \%)$ & $24(88.9 \%)$ & .622 \\
\hline African American & $1(3.6 \%)$ & $2(4.4 \%)$ & .855 & $2(4.3 \%)$ & $1(3.7 \%)$ & .894 \\
\hline Other & $1(3.6 \%)$ & $6(13.3 \%)$ & .168 & $5(10.9 \%)$ & $2(7.4 \%)$ & .628 \\
\hline \multicolumn{7}{|l|}{ Diagnosis } \\
\hline CAD & $26(92.9 \%)$ & $43(93.5 \%)$ & .918 & $45(95.7 \%)$ & $24(88.9 \%)$ & .258 \\
\hline ICM & $2(7.1 \%)$ & $3(6.50 \%)$ & .918 & $2(4.3 \%)$ & $3(11.1 \%)$ & .258 \\
\hline Other & $0(0.0 \%)$ & $0(0.0 \%)$ & 1.000 & $0(0.0 \%)$ & $0(0.0 \%)$ & 1.000 \\
\hline IABP use & $25(89.3 \%)$ & $38(82.6 \%)$ & .434 & $42(89.4 \%)$ & $21(77.8 \%)$ & .178 \\
\hline MI-LVAD interval (d) & $11.1 \pm 16.8$ & $8.8 \pm 11.3$ & .490 & $3.0 \pm 2.3$ & $21.3 \pm 17.1$ & .001 \\
\hline
\end{tabular}

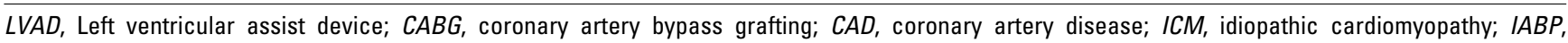
intra-aortic balloon pump; $M I$, myocardial infarction.

compared with that in the subacute MI-LVAD interval group $(P=.013$, Figure 2$)$.

\section{Post-LVAD Survival}

In absolute percentages the 6- and 12-month post-LVAD survivals for the entire study cohort were $67.6 \%$ and $63.5 \%$, respectively. When these rates were evaluated according to revascularization status, both 6- and 12-month post-LVAD survivals were significantly higher in the LVAD group than in the CABG plus LVAD group $(P=.006$, Figure 3$)$.

\section{Postoperative Mortality}

Early ( $\leq 30$ day) mortality was significantly higher in the CABG plus LVAD group compared with that in the LVAD group (18 [39.1\%] patients vs 4 [14.3\%] patients, $P=.020)$

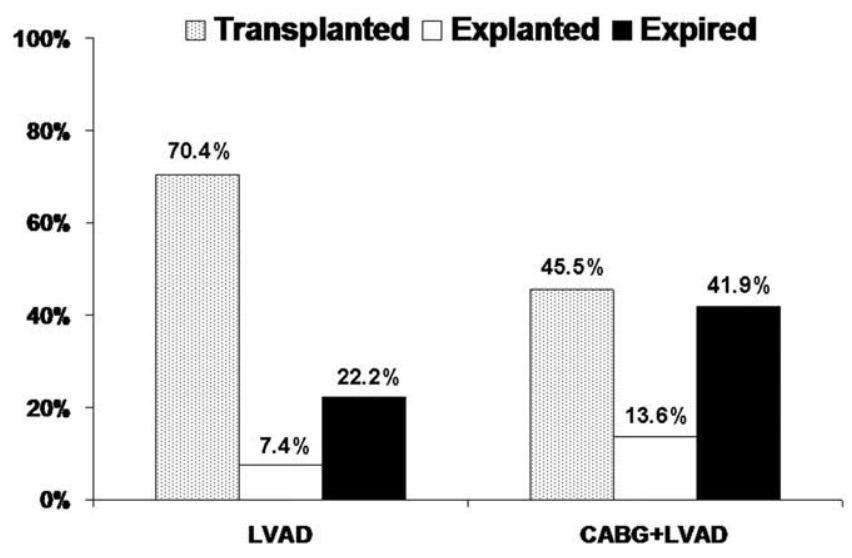

Figure 1. Clinical outcomes of patients receiving LVADs: LVAD versus CABG plus LVAD groups. and in the acute compared with the subacute MI-LVAD interval groups (18 [38.3\%] patients vs 4 [14.8\%] patients, $P=.033$ ).

Overall mortality (early and late) was equivalent between the LVAD and CABG plus LVAD groups $(P=$ .106 , Figure 1) but significantly higher in the acute versus subacute MI-LVAD interval group $(P=.013$, Figure 2$)$.

\section{Duration of LVAD Support}

The mean duration of LVAD support for all patients was $75.4 \pm 91.3$ days. The mean duration of LVAD support up to transplantation, device explantation, or death was $92.5 \pm$ 89.0 days (range, 16-407 days), $62.4 \pm 98.0$ days (range, 4-83 days), and $56.7 \pm 102.3$ days (range, 0-385 days), respectively.

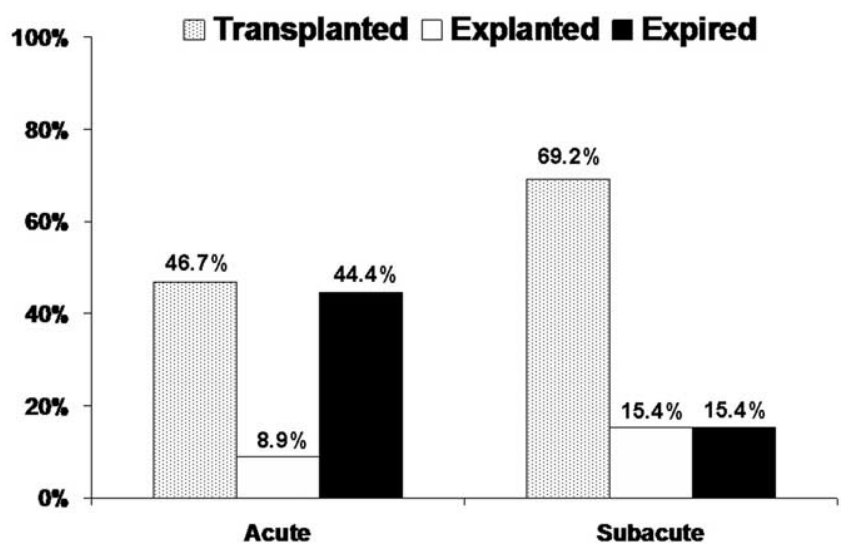

Figure 2. Clinical outcomes of patients receiving LVADs: acute versus subacute MI-LVAD interval groups. 


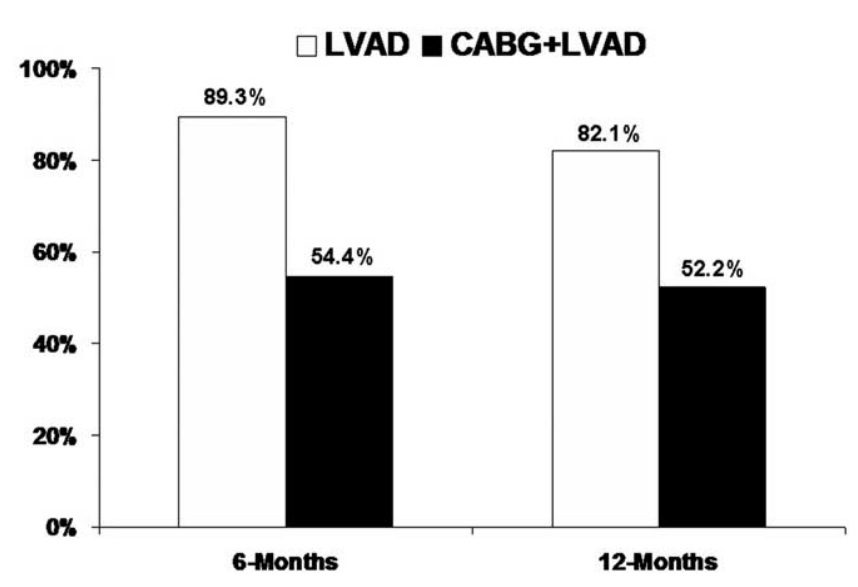

Figure 3. Post-LVAD survivals in the LVAD and CABG plus LVAD groups.

\section{Hospital and ICU LOS}

Total hospital LOS was $60.0 \pm 56.2$ days in the LVAD group and $39.9 \pm 34.8$ days in the CABG plus LVAD group $(P=.353)$. Total hospital LOS was significantly greater in the subacute MI-LVAD interval group than in the acute MI-LVAD interval group $(59.1 \pm 55.9$ vs $34.5 \pm 32.8$ days, $P=.022)$. ICU LOS was similar between the LVAD and CABG plus LVAD groups (14.2 \pm 9.8 vs $17.6 \pm 14.5$ days, $P=.480)$ and between the acute and subacute MI-LVAD interval groups $(17.4 \pm 21.7$ vs $13.9 \pm 9.0$ days, $P=.473)$.

\section{Posttransplantation Survival}

Posttransplantation actuarial survivals at 1, 3, 5, and 7 years in the LVAD and CABG plus LVAD groups were $94.7 \%$, $94.7 \%, 94.7 \%$, and $94.7 \%$ and $79.5 \%, 74.2 \%, 67.1 \%$, and $67.1 \%(P=.066)$, respectively. Survivals in the acute and subacute MI-LVAD interval groups were $85.4 \%, 80.0 \%$, $80.0 \%$, and $80.0 \%$ and $88.9 \%, 88.9 \%, 80.0 \%$, and $80.0 \%(P$ $=.764)$, respectively.

\section{Discussion}

Acute MI complicated by CS remains a highly morbid disease entity. The SHOCK (SHould we emergently revascularize Occluded Coronaries for cardiogenic shocK?) trial is the only randomized controlled clinical study that has evaluated the efficacy of revascularization (through PCI or $\mathrm{CABG}$ ) versus conventional medical therapy in the treatment of CS complicating acute $\mathrm{MI}^{2}$ Although emergency revascularization did not significantly reduce 30-day mortality, after 6 months, there was a significant survival benefit with revascularization compared with medical therapy, an advantage that extended to 1 year. Nonetheless, these survivals were mediocre, at best $49.7 \%$ at 6 months and $46.7 \%$ at 1 year.
These survival outcomes prompted the present study, which sought to determine whether LVAD insertion might be considered as primary therapy for acute MI and CS in lieu of revascularization. Numerous design modifications and adjustments in perioperative management have been applied to LVADs as a result of past clinical experience, such that bridge-to-transplantation success rates of $60 \%$ to $73 \%$ are now commonly reported. ${ }^{16-19}$ Such improvements in survival with the judicious application of LVADs might similarly translate to the acute MI and CS population.

In this study, patients who had acute anterior wall MI complicated by $\mathrm{CS}$ and subsequently underwent direct LVAD implantation had significantly higher 6- and 12month post-LVAD survivals than those who underwent attempted revascularization with $\mathrm{CABG}$ before eventually requiring $L V A D$ support. These findings are curious in light of observations by other studies that patients referred for revascularization, either $\mathrm{PCI}$ or $\mathrm{CABG}$, are generally younger and healthier. ${ }^{20-22}$ They suggest that despite the fact that select patients with acute MI and CS might be clinically stable enough to undergo revascularization by way of $\mathrm{CABG}$, when these patients ultimately require LVAD support, the additional CABG intervention might confer heightened post-LVAD mortality.

The overall 6- and 12-month post-LVAD survivals for the entire LVAD population in this study were $67.6 \%$ and $63.5 \%$, respectively. For the CABG plus LVAD group, these rates were $54.4 \%$ and $52.2 \%$, respectively. Therefore, even the more ill-fated of the 2 LVAD cohorts, and certainly the LVAD population as a whole, achieved higher survivals than did the early revascularization group in the SHOCK trial (49.7\% and $46.7 \%$, respectively). Although this comparison pits the results of an observational study against those of a prospective, randomized clinical trial, the differences in survival are compelling. These findings suggest that the application of LVAD support at any point in the treatment course for acute MI and CS improves survival, regardless of any attempts toward revascularization. The exclusion of revascularization through CABG would seem to heighten survival even further.

The higher early mortality rate in the acute versus subacute MI-LVAD interval group portrays the fact that the acute group represents a sicker cohort of patients. Indeed, it is likely the severity of CS in these patients that prompts immediate LVAD implantation. The greater incidence of early mortality within this group probably represents individuals dying of their illness compounded by surgical intervention rather than the development of complications related to surgical intervention alone.

Posttransplantation survivals between the LVAD and CABG plus LVAD groups and between the acute and subacute MI-LVAD interval groups were equivalent. However, the trend toward lower posttransplantation survival in 
the CABG plus LVAD group intimates that the post-LVAD systemic milieu in this group might be persistently compromised in such a way that carries into the posttransplantation state.

Although this study suggests that CABG alone confers poorer survival than LVAD as primary therapy for acute MI and CS, the question might arise whether patients might fare best with simultaneous CABG and LVAD. The LVAD can facilitate unloading of the heart in the critical perioperative phase to minimize the work performed by the myocardium. Conversely, the utility of CABG and its associated prolonged operative and cardiopulmonary bypass times is debatable because most of these patients undergo transplantation anyway.

\section{Limitations of the Study}

The limitations of this study are those inherent with retrospective analyses. Selection bias is evident because this study fails to account for the probable vast number of patients in similar clinical scenarios who undergo revascularization and require no further intervention. Therefore, the patients in the CABG plus LVAD group likely represent a particularly sick cohort of individuals whose shock was not ameliorated by revascularization alone. Data on the proportion of these patients who presented with failed grafts are lacking.

The definition of acute MI as having occurred 3 months or less before LVAD insertion was established to ensure thorough inclusion of all qualified patients for this study. This broad inclusion interval likely introduces 2 distinct entities of patients with acute MI and CS who might arguably be managed differently. However, it is often difficult to predict and discern truly sick patients from those slightly less sick on the basis of acute MILVAD interval alone, and we did not want to exclude from our analysis those requiring later LVAD insertion.

Revascularization by way of PCI is not represented in this study when, in fact, the majority of revascularization efforts are carried out with this approach. CABG often becomes an option only after PCI has failed or is not feasible, again emphasizing the relative sickness of this group of patients. Unfortunately, data reporting the frequency of failed PCI attempts referred for CABG are not available in this study.

\section{Conclusions}

Patients with acute anterior wall MI complicated by CS who undergo revascularization through $\mathrm{CABG}$ only to subsequently require LVAD support have lower postLVAD survivals than those in a similar clinical setting who undergo direct LVAD implantation. The decisionmaking processes that underlie patient selection and determination of therapeutic intervention need to be eluci- dated. Ultimately, confirmation of the findings in this study would require conducting a large, multicenter, randomized clinical trial comparing revascularization (PCI or CABG) versus LVAD support as primary therapy for acute MI complicated by CS.

\section{References}

1. Hochman JS, Sleeper LA, White HD, Dzavik V, Wong SC, Menon V, et al. SHOCK Investigators. Should We Emergently Revascularize Occluded Coronaries for Cardiogenic Shock. One-year survival following early revascularization for cardiogenic shock. JAMA. 2001; 285:190-2.

2. Hochman JS, Sleeper LA, Webb JG, Sanborn TA, White HD, Talley JD, et al. Early revascularization in acute myocardial infarction complicated by cardiogenic shock. SHOCK Investigators. Should We Emergently Revascularize Occluded Coronaries for Cardiogenic Shock. N Engl J Med. 1999;341:625-34.

3. Lee L, Bates ER, Pitt B, Walton JA, Laufer N, O’Neill WW. Percutaneous transluminal coronary angioplasty improves survival in acute myocardial infarction complicated by cardiogenic shock. Circulation. 1988;78:1345-51.

4. Lindholm MG, Aldershvile J, Sundgreen C, Jorgensen E, Saunamaki $\mathrm{K}$, Boesgaard S. Effect of early revascularisation in cardiogenic shock complicating acute myocardial infarction. A single center experience. Eur J Heart Fail. 2003;5:73-9.

5. Dauerman HL, Goldberg RJ, White K, Gore JM, Sadiq I, Gurfinkel E, et al. Global Registry of Acute Coronary Events. GRACE Investigators. Revascularization, stenting, and outcomes of patients with acute myocardial infarction complicated by cardiogenic shock. Am J Cardiol. 2002;90:838-42.

6. Webb JG, Sanborn TA, Sleeper LA, Carere RG, Buller CE, Slater JN, et al. SHOCK Investigators. Percutaneous coronary intervention for cardiogenic shock in the SHOCK Trial Registry. Am Heart J. 2001; 141:964-70.

7. Eltchaninoff H, Simpfendorfer C, Franco I, Raymond RE, Casale PN, Whitlow PL. Early and 1-year survival rates in acute myocardial infarction complicated by cardiogenic shock: a retrospective study comparing coronary angioplasty with medical treatment. Am Heart J. 1995;130:459-64.

8. Moosvi AR, Khaja F, Villanueva L, Gheorghiade M, Douthat L, Goldstein S. Early revascularization improves survival in cardiogenic shock complicating acute myocardial infarction. J Am Coll Cardiol. 1992;19:907-14.

9. Webb JG, Lowe AM, Sanborn TA, White HD, Sleeper LA, Carere RG, et al. SHOCK Investigators. Percutaneous coronary intervention for cardiogenic shock in the SHOCK trial. J Am Coll Cardiol. 2003;42: 1380-6.

10. Rose EA, Gelijns AC, Moskowitz AJ, Heitjan DF, Stevenson LW, Dembitsky W, et al. Randomized Evaluation of Mechanical Assistance for the Treatment of Congestive Heart Failure (REMATCH) Study Group. Long-term mechanical left ventricular assistance for end-stage heart failure. N Engl J Med. 2001;345:1435-43.

11. Berger RL, Merin G, Carr J, Sossman HA, Bernhard WF. Successful use of a left ventricular assist device in cardiogenic shock from massive postoperative myocardial infarction. J Thorac Cardiovasc Surg. 1979;78:626-32.

12. Park SJ, Nguyen DQ, Bank AJ, Ormaza S, Bolman RM 3rd. Left ventricular assist device bridge therapy for acute myocardial infarction. Ann Thorac Surg. 2000;69:1146-51.

13. Hendry PJ, Masters RG, Mussivand TV, Smith S, Davies RA, Finlay $\mathrm{S}$, et al. Circulatory support for cardiogenic shock due to acute myocardial infarction: a Canadian experience. Can J Cardiol. 1999;15: 1090-4.

14. Chen JM, DeRose JJ, Slater JP, Spanier TB, Dewey TM, Catanese KA, et al. Improved survival rates support left ventricular assist device implantation early after myocardial infarction. J Am Coll Cardiol. 1999;33:1903-8. 
15. Moritz A, Wolner E. Circulatory support with shock due to acute myocardial infarction. Ann Thorac Surg. 1993;55:238-44.

16. Morgan JA, John R, Rao V, Weinberg AD, Lee BJ, Mazzeo PA, et al. Bridging to transplant with the HeartMate left ventricular assist device: the Columbia Presbyterian 12-year experience. J Thorac Cardiovasc Surg. 2004;127:1309-16.

17. Frazier OH, Delgado RM. Mechanical circulatory support for advanced heart failure: where does it stand in 2003? Circulation. 2003; 108:3064-8.

18. Frazier OH, Rose EA, McCarthy P, Burton NA, Tector A, Levin H, et al. Improved mortality and rehabilitation of transplant candidates treated with a long-term implantable left ventricular assist system. Ann Surg. 1995;222:327-38.

19. Robbins RC, Kown MH, Portner PM, Oyer PE. The totally implantable Novacor left ventricular assist system. Ann Thorac Surg. 2001; 71(suppl):S162-5; discussion S183-4.
20. Hochman JS, Boland J, Sleeper LA, Porway M, Brinker J, Col J, et al. Current spectrum of cardiogenic shock and effect of early revascularization on mortality. Results of an International Registry. SHOCK Registry Investigators. Circulation. 1995;91:873-81.

21. Berger PB, Holmes DR Jr, Stebbins AL, Bates ER, Califf RM, Topol EJ. Impact of an aggressive invasive catheterization and revascularization strategy on mortality in patients with cardiogenic shock in the Global Utilization of Streptokinase and Tissue Plasminogen Activator for Occluded Coronary Arteries (GUSTO-I) trial. An observational study. Circulation. 1997;96:122-7.

22. Berger PB, Tuttle RH, Holmes DR Jr, Topol EJ, Aylward PE, Horgan $\mathrm{JH}$, et al. One-year survival among patients with acute myocardial infarction complicated by cardiogenic shock, and its relation to early revascularization: results from the GUSTO-I trial. Circulation. 1999; 99:873-8.

\section{JTCVS On-Line Manuscript Submission and Review}

The Journal of Thoracic and Cardiovascular Surgery requires authors and reviewers to submit all new and revised manuscripts and reviews via Editorial Manager. Point your browser to http://jtcvs.editorialmanager.com, log in as author or reviewer (as appropriate), and follow the instructions provided.

To retrieve your username and password, click "Forget your password?" on the Editorial Manager log-in page.

If you have questions or experience problems uploading your manuscript or review, please contact the editorial office:

Telephone: 215-762-1854

E-mail: jtcvs@drexel.edu 